\title{
Researches on the design of customized femoral implant
}

\author{
Dan Leordean ${ }^{1}$, Tatiana Ciobanu ${ }^{1, *}$ and Mircea Rusu ${ }^{1}$ \\ ${ }^{1}$ Technical University of Cluj-Napoca, Romania
}

\begin{abstract}
Innovations in the industry have also proved to be quite impressive in the medical field, where another approach is needed due to the need for personalization. Due to femoral fractures, it is not just enough to stabilize the bone, but also to have the integration of the implant with the host bone. This research is intended to undertake studies on the redesign of the distal femoral plate. The redesign had been elaborated to increase the number of people who are compatible with this type of femoral plate and also, to improve the physico-mechanical and biological properties toward to a commercial distal femoral plate made of type $316 \mathrm{~L}$ stainless steel. Within SolidWorks software, a static simulation has been run after there have been defined restraints, external loads, and a mesh. The parameters were similar to those after the implantation. Taking this reason into consideration, the final results will be improved by modifying plate's material from $316 \mathrm{~L}$ to titanium alloy Ti6Al7 Nb to increase the capability and biocompatibility. Moreover, the geometry of the distal femoral plate changes to decrease the weight and time of osteosynthesis. The final implant is parametrized 3D models that handle all day-to-day activities and has a weight $50 \%$ lower than that of the commercial implant.
\end{abstract}

\section{Introduction}

A fracture represents a broken bone or a bone discontinuity. It can be broken completely or partially fractured in different ways: in length, oblique or in the cross. The reasons for a bone fracture are trauma, osteoporosis or excess of physical effort. The trauma can be a fall, a car accident, a motorcycle accident, or an accident during a sport: a tough football game. Another reason for favoring bone fractures is osteoporosis - a disease that diminishes the bone tissue density and increases the fragility of the bone. In addition to the reasons presented, another cause of bone fracture is the excess of physical effort [1][2]. Fractures due to this reason are found in athletes who get their muscles tired by repetitive movements and put pressure on their bones. Fractures from this cause are stress fractures.

The fracture of the femur is a rupture of the femoral bone, a rupture that is along the long and straight part of the bone, called femoral diaphysis. The femur can also fracture in the upper and lower parts of the femur. Symptoms of a fracture of the femur are: sharp and instant pain, impossibility of moving and maintaining your own weight on the foot, shortening of injured leg, deformation of fractured area [3].

\footnotetext{
*Corresponding author: tania.ciobanu96@gmail.com
} 
An orthopedic implant is a medical device that replaces a missing link, a bone, or sustains a fractured bone. Osteosynthesis plates are plates of different configurations and sizes, with holes of various types designed to fix and strengthen a femoral fracture together with specially made screws, mounted perpendicularly to the axis of the bone. Blocked plates are implants with fixed angles, which leads to locking the bolts and preventing the movement. In addition to stable fixation, blocked plates also have the advantage of minimally invasive technique - due to the implantation method the destruction of any tissue is minimal [3]. To confirm the biocompatibility of the implant with a body, both the implant material and the body must not cause reactions. Biomaterial is a synthetic material used to replace or support a part of a living system. Based on the origin of biomaterials there are two categories: natural biomaterials and synthetic biomaterials. Natural proteins include proteins such as collagen, protein fibers - silk, leather, wool, metals such as titanium, nickel, magnesium, and composite nonmetals - Kevlar. Synthetic biomaterials are alloys of metals, non-metals, ceramic materials and composite materials - carbon fiber. Among the metals found in the implants of any kind are: stainless steel, titanium, nickel, zirconium [4].

The direct method of implant generation has the following steps: computer tomography, visualization of the affected zone, 3D implant design, implant manufacturing using SLM technology, final implant adjustment. Due to advanced technology, SLM is the best alternative for implant manufacturing [2].

Materials are tested in order to become biomaterials and there are a few requirements to be met: biocompatibility, resistance to corrosion, suitable porosity and roughness, acceleration of osteosynthesis.

\section{Methods and materials}

\subsection{Methods}

Due to the lack of a free 3D model of femoral distal plate offered by medical implants manufacturers or suppliers, we have appealed to images published on the websites of the companies they offer for marketing. Designing the 3D model of the implant we completed a few stages:

- With the high-quality images, technical specifications and details, we have succeeded in 3D modeling of the distal femoral plate distributed by Biomatrix System [5]. The number of holes is taken according to the total length of the distal plate. We chose 169.3 $\mathrm{mm}$ for length for a medium height person, and 4 holes. After analyzing the drawing, we imported it into SolidWorks to start the design itself.

- We use SolidWorks software to design the femoral plate due to its versatility and multitude of features. Enter the image in which the lateral view is represented in the right plane. Using the image conversion function in the sketch, we draw the curve that generates the femoral implant lateral profile, fig. 1, a).

- We created an open surface from this contour, fig. 1, b). We introduced the image with side view representation in the front plane and repeated the conversion image function to create the curve that generates the frontal profile of the distal femoral plate, fig. $1 \mathrm{a}$ ). We intersect the resulted complex surfaces, fig. 1, c) and created a solid object that will have a complex geometry. We used the surface cutting function to result a final solid body.

- The holes of $\varnothing 5 \mathrm{~mm}, \varnothing 2.5 \mathrm{~mm}, \varnothing 3 \mathrm{~mm}$, and the slot are represented by creating a minimum $5 \mathrm{~mm}$ plan above the plate. Then drill it with the Cut -Extrude function. 
- The next step is to make the holes of the hole and edge of the Chamfer plate embedded in the SolidWorks software. The chamfers have different dimensions because of the complex surface they are part of. The final model is shown in fig. $1 \mathrm{~d}$ ).

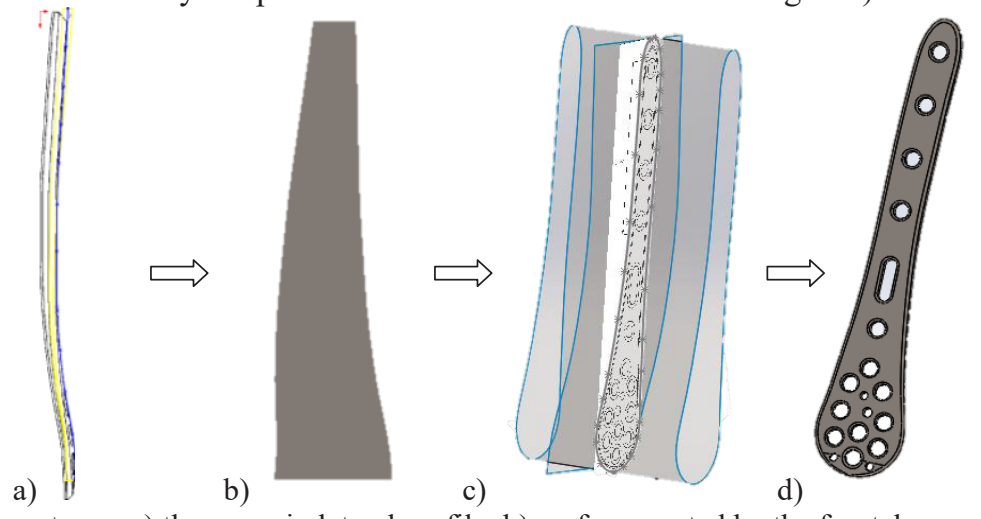

Fig. 1. Design stages: a) the curve in lateral profile; b) surface created by the frontal curve; c) intersect surfaces, d) final femoral plate.

Using the surface functions present in the program, we succeeded in creating the virtual model similar to the Biomatrix System. Following modeling and material properties definition, the commercial femoral distal plate has $118 \mathrm{~g}$.

In this part we performed the finite element analysis of the distal femoral plate, a commercial model, using SolidWorks 2016 design software. The plate was made of stainless steel. For finite element analysis the same software was used. The 3D model has been entered into the Finite Element Analysis module of the program, and data parameters for the material have been set. A foot implant must withstand up to 1.5 times the weight of the body to sustain in walking time [6] and up to 8 times in running. Considering this, implant analysis will be done by loading it at $4000 \mathrm{~N}$, which means $8 \times 50 \mathrm{~kg}$ (patient's weight). $4000 \mathrm{~N}$ is selected to observe the behavior of the femoral plate during a limiting event. To meet all requirements, the femoral plate must withstand all normal and unfavorable conditions [7], and to illustrate the results of the analysis, four studies with different parameters were performed.

\subsection{Materials}

The difference between the two materials used in finite element analysis is made by the physical and mechanical properties each one possesses. The properties that help to achieve the main purpose of the implant are illustrated in Table 1.

Table 1. Mechanical properties of stainless steel 316L type [8] and titanium alloy Ti6 Al7 Nb [9].

\begin{tabular}{|c|c|c|c|c|c|}
\hline Grade & $\begin{array}{c}\text { Tensile strength } \\
{[\mathrm{MPa}]}\end{array}$ & $\begin{array}{c}\text { Yield Strength } \\
{[\mathrm{MPa}]}\end{array}$ & $\begin{array}{c}\text { Elongation } \\
{[\%]}\end{array}$ & $\begin{array}{c}\text { Poisson's } \\
\text { Ratio }\end{array}$ & $\begin{array}{c}\text { Density } \\
{\left[\mathrm{g} / \mathrm{cm}^{3}\right]}\end{array}$ \\
\hline $316 \mathrm{~L}$ & 485 & 170 & 40 & 0.265 & 8 \\
\hline $\begin{array}{c}\text { Ti6A17Nb } \\
(100 \mathrm{~W})\end{array}$ & 400 & 400 & 10 & 0.36 & 4.34 \\
\hline
\end{tabular}

\subsubsection{Stainless steel 316L}

Steel is an alloy that contains iron and less than 2.2\% carbon. Depending on the carbon content, there is the type and characteristics of the steel. In the chemical composition of stainless steel, 
carbon occupies a small amount, but chromium is the element that makes it resistant to corrosion - at least $11 \%$ chromium. This property is due to the creation of a thin layer of chromium oxide that does not allow corrosion and has self-healing properties. The material used in the manufacture of distal femoral osteosynthesis plate is $316 \mathrm{~L}$ stainless steel or X2CrNiMo17- 12 2. It's molybdenum - rich stainless steel. It has a higher corrosion resistance than the classic nickel - chromium 302-304 stainless steel alloy. 316L type stainless steel has good resistance to high temperatures, corrosion resistance and high tensile strength, properties shown in table 1.

We performed different analyses with finite elements to observe the behavior of the material. The input data of the finite element analysis were different and illustrated the parameters in an ordinary and abnormal situation [10]. An ordinary situation is characterized by a uniform loading with $4000 \mathrm{~N}$ on 4 holes that are located on the femur and the fixed geometry is set on chamfers of the 6 fixing holes. The analysis for this situation is shown in fig. 2, a).

For an abnormal situation the data entered were:

- Defined material: 316L stainless steel or X2CrNiMo17-12-2;

- Load on 3 holes that after implantation will be located to the knee area;

- The loading is $4000 \mathrm{~N}$ and is along the longitudinal axis of the femur;

- The fixed geometry is set on chamfers of the two fixing holes, which after implantation are on the femoral component;

- The movements in the transverse direction to simulate the contact with the femur are represented with an advanced fixture.

The results of this study are illustrated in figure 2, b) where you can observe that the von Mises maximum value is exceeding the maximum allowed value $170 \mathrm{MPa}$.

a)

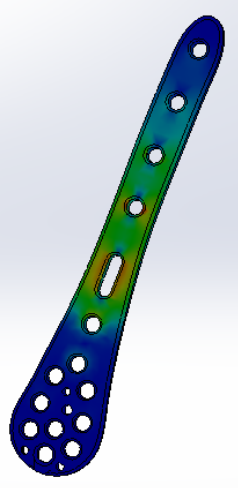

b)

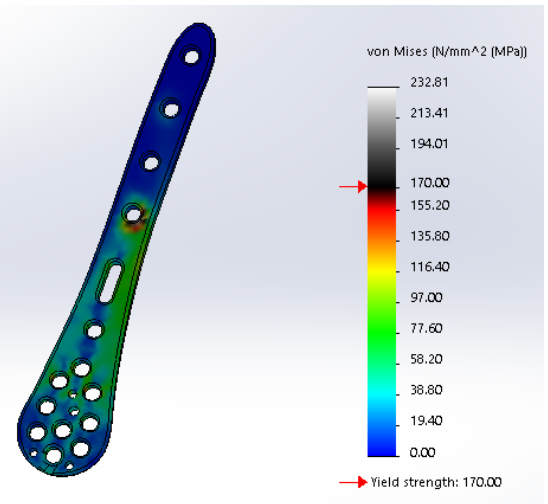

Fig. 2. Stress von Mises: a) normal situation parameters b) abnormal parameters.

\subsubsection{Titanium alloy}

The Ti-6Al-7Nb alloy is an alloy used in medicine, mostly for the manufacture of prostheses and implants, for example: fracture fixation plates, intermediate rods and nails, screws and medical threads. Titanium alloys have many advantages including titanium resistance is higher than stainless steel, the weight of titanium is lower than that of stainless steel, titanium has a high resistance to repeated loads, making it ideal for medical applications. Titanium and its alloys are the most common biomaterials due to biocompatibility, a problem that it solves because of properties very close to those of the bone: corrosion resistance, modulus of elasticity. As well as the source [10], implants made of titanium alloy or pure titanium have a thin layer of $\mathrm{TiO}_{2}(3-8$ $\mathrm{nm})$. The layer is responsible for osseointegration - the ability to form a direct and functional contact of the implant with bone tissue. In order to monitor the stress state and the influence of the material on it, we chose to perform a finite element analysis on the distal femoral plate, the 
commercial model, but to which we modify the mechanical properties by adding the properties of the titanium alloy Ti6Al7Nb obtained by SLM technology using $100 \mathrm{~W}$ laser power [1]. As a result of the UTC-N research, it is established that the titanium alloy is a very good variant for the implant manufacturing because of the great similarities between the elastic and mechanical properties of the material with the bone, especially the material whose structures are made with the power of the $100 \mathrm{~W}$ laser beam. Steps are similar to first analyses and the parameters were:

- Defined material: Ti6Al7Nb (100W);

- The implant is loaded onto 4 holes which after implantation are on the femoral component;

- The load is $4000 \mathrm{~N}$ and is along the long axis of the femur;

- Fixed 6-hole knee attachment.

a)

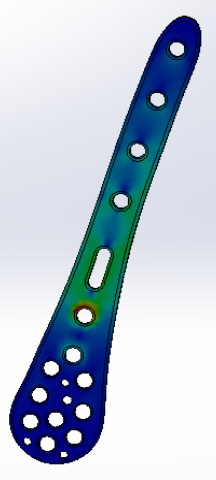

b)

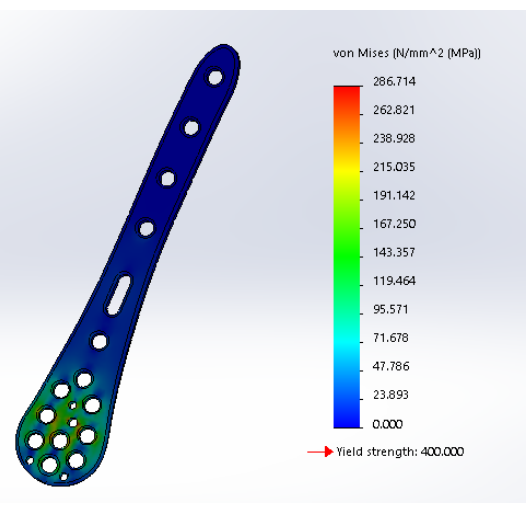

Fig. 3. Commercial plate - Titanium alloy: a) normal parameters, b) abnormal parameters.

As shown in figure 3, the implant made of titanium alloy has a better mechanical behavior than that made of stainless steel alloy in both normal and abnormal situations.

\section{Results and Discussion}

Based on the finite element analyzes performed on the femoral plate, the overloaded areas were identified and the geometry and material changed to improve the requirements that an implanted medical device must meet.

The implant redesigning has many advantages. One of them is that working time decreases due to decreased processing volume. The complexity of geometry is not a disadvantage [1], because the SLM process can perform any kind of geometry.

To sum up all these advantages that the final model (fig. 4, d) of the femoral implant has, we have redesigned several variants (fig. 4) with different geometries. The final criterion for choosing the last model was the analysis with finite elements (fig. 5) for an abnormal case that illustrates that the implant can fulfill its purpose even in unusual situations [11].
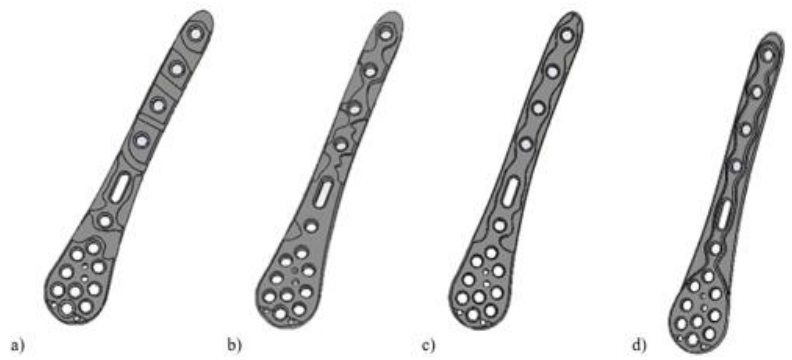

Fig. 4. Different models for redesign. 
a)

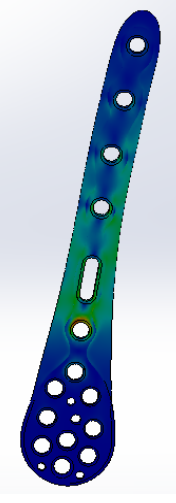

b)

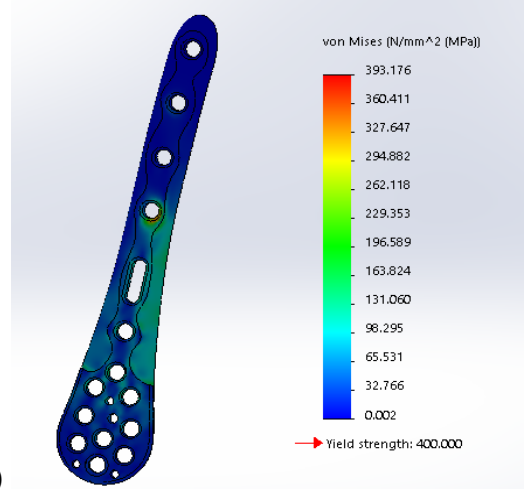

Fig. 5. von Mises stress - Redesigned femoral plate. a) normal parameters; b) abnormal parameters.

\section{Conclusions}

The main objective was achieved with the modeling and the analysis with finite elements of a commercial model of the femoral plate, the design of a distal femoral plate with improved characteristics and the preparation for the manufacture of the plate designed. It was possible to model the distal femoral plate and to subject it to normal and unfavorable conditions. We established that the femoral plate needs to be improved by removing the material and increasing the mechanical properties. This was achieved by modifying the stainless steel in a titanium alloy Ti6Al7Nb. The projected femoral distal plate has a high biocompatibility compared to that of stainless steel. In addition, the weight of the new plate decreased by $50 \%$ by removing the material from the passive surface. The new distal femoral plate has a pleasant, organic and symmetrical appearance. The thickness of the plate decreased by $\sim 40 \%$ and varies between 3-6 mm. Another advantage that comes with plate design is the distribution of equivalent stress. Due to the redesign and modification of the material, the implant has a higher resistance, and the stress graph indicates that the plate has a higher limit.

This work was supported by a grant of Romanian Ministry of Research and Innovation UEFISCDI, project number PN-III-P1-1.2-PCCDI-2017-0224, Contract 77PCCDI/2018 within PNCDI III program.

\section{References}

1. P. Berce, N. Bâlc, D. Leordean, et al., Romanian Acad. Publish., ISBN 9789732725917, (2015)

2. N. Balc, S.C. Cosma, M. Moldovan, P. Berce, Chap. 2. Medical applications of macro-porous structures additive manufactured, Book: Adv. ind. eng. - New tend. in mat. Eng., pp.47-64, (2017)

3. www.ortovit.eu/ortopedie/trauma/placi conventionale.html

4. S.C. Cosma, D. Leordean, Balneo-Research Journal, Vol. 6 (4), (2015)

5. https://biomatrixsystem.ro

6. G. Bergmann, Deuretzbacher G., Heller M., Graichen F., Rohlmann A., et al, J Biomech., (2001)

7. K. Utku, Distal femur: dynamization of plating, Injury, Vol. 49, Supp. 1, (2018)

8. www.lenntech.com/stainless-steel-3161.htm\#ixzz5sLakNR6p

9. M. Basiaga, W. Kajzer, W. Walke, A. Kajzer, M. Kaczmarek, Eval. of phys-chem. Prop. of surf. Modif. Ti6Al4V and Ti6Al7Nb alloys used for orthp. implants, Mat. Sci. and Eng.: Vol. 68, (2016) 10. A. Dhanopia, M. Bhargava, Procedia Engineering, Finite Element Analysis of Human Fractured Femur Bone Implantation, Vol. 173, 2017

11. W. Hoskins, Ro. Bingham, X. Griffin, Dist. Fem. fract. in ad., Orth. \& Trauma, Vol. 31 (2), 2017 